\title{
Magnetohydrodynamic effects on finite and infinite slider bearings
}

\author{
M. MOUDA ${ }^{1}$ M. NABHANI ${ }^{2}$, M. EL KHLIFI ${ }^{3}$
}

\author{
Hassan II University of Casablanca, Faculty of Sciences and Techniques \\ PO Box 146, 20650 Mohammedia, Morocco \\ ${ }^{1}$ mouhcine.mouda-etu@etu.univh2c.ma \\ ${ }^{2}$ nabhanimohamed@yahoo.fr \\ ${ }^{3}$ mohmed.elkhlifi@fstm.ac.ma
}

\begin{abstract}
This paper presents a numerical investigation of lubricating slider bearings with conducting couple stress fluids using externally applied magnetics fields. The modified two-dimensional magnetohydrodynamic couple stress Reynolds-type equation is obtained. This governing equation is resolved numerically by using finite difference scheme, which involves the GaussSeidel method to compute the bearing characteristics. Numerical results using different considered values of the couple stress and Hartman number are presented. These results demonstrate that the transverse magnetic field and couple stress effects are significant.
\end{abstract}

\section{Keywords:}

Magnetohydrodynamics; Stokes microcontinuum theory; Couple Stress; Slider Bearing

\section{Introduction}

In recent years, the study of magneto-hydrodynamic (MHD) lubrication of bearings has attracted the attention of several researchers from various fields, particularly researchers from the field of nuclear and space engineering. The MHD bearings with conducting fluids possess numerous advantages over the conventional bearings. Having an appreciably increased load capacity due to the MHD effects, it is also able to function under high temperature. Many authors have investigated the magneto-hydrodynamic (MHD) performance of slider bearings ${ }^{1-6}$. In this paper, a numerical investigation of the electric conducting behaviour of non-Newtonian lubricants confined in slider bearings is presented. The non-Newtonian behaviour of the lubricant is described using the micro-continuum Stokes theory ${ }^{7}$. The governing partial differential equations are discretized by finite differences. The resulting algebraic equations are solved using Gauss Seidel method. Numerical results of the load capacity are presented and commented.

\section{Governing equations and numerical resolution}

This numerical study concerns the viscous flow of an electrically conducting fluid confined between two plane surfaces. The lower surface, coinciding with the plane $z=0$, is moving with a constant sliding velocity $U$ in the $x$-direction. The upper surface is inclined, fixed. The lubricant is taken to be an isothermal, incompressible, electrically conducting, and non-Newtonian couple stress fluid of constant properties. It is assumed that the body forces and body couples are negligible except for the Lorentz force. The induced magnetic field is neglected compared with the imposed magnetic field $\vec{B}_{0}$, which is uniform and oriented in the $z$-direction. Based upon the assumption of thin film, the dimensionless governing equations for laminar steady viscous flow in the presence of a stationary magnetic field can be written as:

$$
\frac{\partial}{\partial x^{*}}\left(F^{*} \frac{\partial p^{*}}{\partial x^{*}}\right)+\frac{1}{\beta^{2}} \frac{\partial}{\partial y^{*}}\left(G^{*} \frac{\partial p^{*}}{\partial y^{*}}\right)=6 \frac{\partial h^{*}}{\partial x^{*}}
$$

with:

$$
\begin{aligned}
& F^{*}=\frac{6}{M^{2}}\left(-2 h^{*}+\frac{1}{l^{*}} \frac{\left(A_{I}^{* 2}-B_{I}^{* 2}\right) h^{* 2}}{\frac{A_{I}^{* 2}}{B_{I}^{*}} \tanh \left(\frac{B_{I}^{*} h^{*}}{2 l^{*}}\right)-\frac{B_{I}^{* 2}}{A_{I}^{*}} \tanh \left(\frac{A_{I}^{*} h^{*}}{2 l^{*}}\right)}\right) \\
& G^{*}=\frac{G}{h_{o}^{3}}=h^{* 3}-12 l^{* 2} h^{*}+24 l^{* 3} \tanh \left(\frac{h^{*}}{2 l^{*}}\right)
\end{aligned}
$$

where $p^{*}$ is the film pressure, $l^{*}=\sqrt{\frac{\eta}{\mu}} / h_{o}$ is the couple stress parameter, $M$ represents the Hartmann number defined by $M=B_{0} h \sqrt{\sigma / \mu}, h_{o}$ is the outlet film thickness, $\sigma$ is the electrical conductivity of the fluid, $\mu$ is the dynamic viscosity coefficient and $\eta$ is the material constant of couple stress fluids.

For plane inclined slider bearings, the dimensionless film thickness is:

$h^{*}=1+\alpha\left(1-x^{*}\right)$

where $a$ is the profile parameter, $\alpha=\frac{h_{i}-h_{o}}{h_{o}}$, here $h_{i}$ is the inlet film thickness.

Equation 1 is discretised by finite difference method using the second-order centred scheme. The algebraic 
equations obtained are iteratively resolved by the technique of successive over-relaxation. The convergence of the numerical scheme is obtained when the relative criterion $\left|\frac{p_{i, j}^{* N_{\text {ITER }}+1}-p_{i, j}^{* N_{\text {ITER }}}}{p_{i, j}^{* N_{\text {ITER }}}}\right| \leq 10^{-6}$ is satisfied. Once the convergence is achieved, both friction force and load capacity are evaluated using rectangular method and Trapeze's rule, respectively.

\section{Results}

The characteristics of magnetohydrodynamic slider bearings using couple stress lubricants and transverse magnetic fields are analysed based on three dimensionless parameters, namely, the Hartmann number and the parameter of couple stress. For both slider bearings types (finite and infinite). It is shown that the couple stress effect increases load capacity (see Fig. 1) but decreases the friction coefficient because the additives will resist and thus oppose the lubricant fluid motion and as consequence enhance the film pressure.

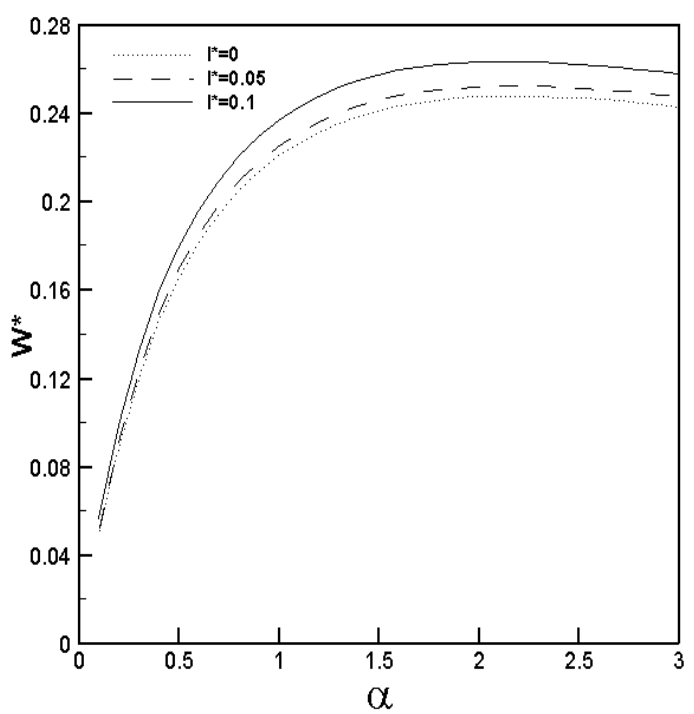

Figure 1. Dimensionless load capacity $W^{*}$ in infinite slider bearing varying with profile parameter $\alpha$ for different $l^{*}$ with $M=4$.

In addition, the using magnetic field increases both load capacity and friction coefficient (see Fig. 2). Moreover, the maximum load capacity and the maximum friction coefficient augment when the bearing is wide. These findings can be useful to provide information for engineers in designing slider bearings.

\section{Conclusion}

This numerical study focuses of MHD plane inclined slider bearings including non-Newtonian couple stress effects. It's shown that the combined influence of MHD,

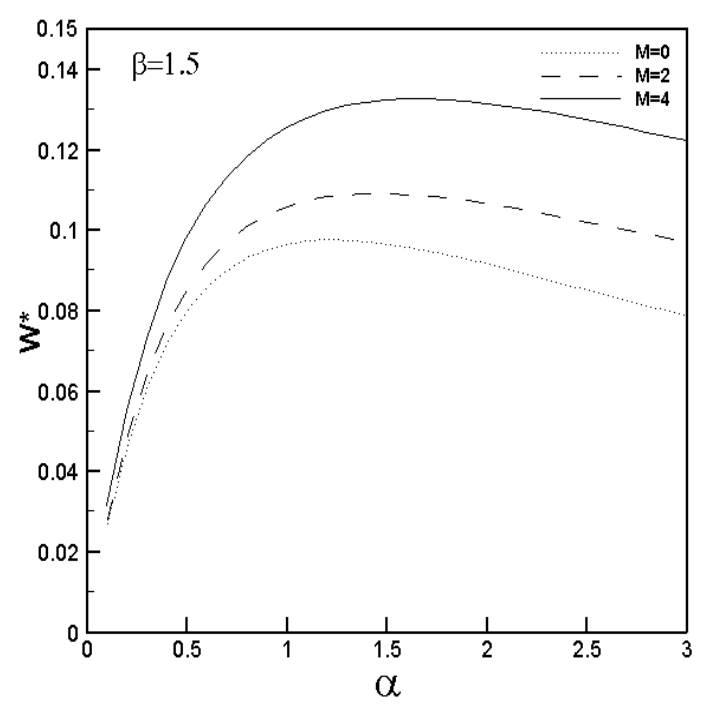

Figure 2. Dimensionless load capacity $W^{*}$ in finite slider bearing varying with profile parameter $\alpha$ for different $M$ with $l^{*}=0.1$.

couple stress on the slider bearing performance are significant and cannot be overlooked.

\section{Références}

[1] J.R. Lin, C.R. Hung, C.H. Hsu, and C. Lai, Dynamic Stiffness And Damping Characteristics Of One-Dimensional MagnetoHydrodynamic Inclined-Plane Slider Bearings, Proceedings of the Institution of Mechanical Engineers, Part J: Journal of Engineering Tribology, 223 (2009) pp. 211-219.

[2] J.R. Lin, and R.F. Lu, Dynamic Characteristics For Magneto-Hydrodynamic Wide Slider Bearings With An Exponential Film Profile, Journal of Marine Science and Technology, 18 (2010) pp. 268-276.

[3] J.R. Lin, MHD Steady And Dynamic Characteristics of Wide Tapered-Land Slider Bearings, Tribology International, 43, pp. (2010) 2378-2383.

[4] J.R. Lin, Dynamic Characteristics For Wide Magneto-Hydrodynamic Slider Bearings With A Power-Law Film Profile, Applied Mathematical Modelling, 36 (2012) pp. 4521-4528.

[5] J.R Lin, R.F. Lu, T.C. Hung, and L.J Liang, Effects Of Magnetic Fields On The Dynamic Characteristics Of Wide Parallel Step Slider Bearings With Electrically Conducting Fluids, Lubrication Science, 24 (2012) pp. 238-250.

[6] M .Nabhani, M. El Khlifi. Inertial MHD couple stress effects on infinitely wide slider bearings. Tribology Transactions, 58 (2015) pp. 374-383.

[7] V.K. Stokes, Couples Stresses In Fluids, Phys Fluids, 9 (1966) pp. 1709-1715. 